\title{
Genética aplicada a la práctica clínica en trastornos del neurodesarrollo
}

\author{
Alberto Fernández-Jaén, Juan C. Cigudosa, Daniel Martín Fernández-Mayoralas, Javier Suela, \\ Ana L. Fernández-Perrone, Beatriz Calleja-Pérez, Sara López-Martín
}

\begin{abstract}
Resumen. Las evidencias genéticas de los trastornos del neurodesarrollo están ampliamente sustentadas en la literatura médica. Los avances en la genética y la tecnología han incrementado la rentabilidad diagnóstica de los estudios actuales de un 3-5\% a un 30-40\% en los pacientes con discapacidad intelectual o trastornos del espectro autista. En este sentido, los estudios por microarrays cromosómicos muestran un mayor poder diagnóstico que las técnicas convencionales (cariotipo, análisis de subtelómeros...). Los protocolos más recientes en el apartado biomédico del estudio genético de estos trastornos sitúan los microarrays cromosómicos como análisis de primera línea, recomendando otros estudios específicos según las características clínicas del paciente (síndrome X frágil, mutación en PTEN...). En la evaluación de otros trastornos del neurodesarrollo (trastorno por déficit de atención/hiperactividad, trastornos del aprendizaje...), la realización de pruebas genéticas está limitada y condicionada a las características clínicas o antecedentes familiares o personales del paciente; incluso en estas situaciones, no existen protocolos de evaluación o derivación genética.
\end{abstract}

Palabras clave. Arrays. Autismo. Discapacidad intelectual. Genética. Trastorno por déficit de atención/hiperactividad. Trastornos del neurodesarrollo.

\section{Introducción}

Los trastornos del neurodesarrollo (TND) son alteraciones o retrasos en el desarrollo del sistema nervioso central, que se manifiestan por disfunciones cerebrales que afectan a la capacidad para el aprendizaje y la competencia social, entre otras aptitudes, impidiendo el normal desarrollo del niño y adolescente. Su propia terminología anticipa tres características relevantes: su origen en la disfunción del sistema nervioso, el componente evolutivo o madurativo y la presencia de manifestaciones clínicas en la infancia (que no exclusiva de esta edad) $[1,2]$. Entre las características básicas de los TND debemos señalar la ausencia de biomarcadores, la presencia de síntomas comunes o similares entre diferentes TND, la frecuente comorbilidad en todos los TND y la compleja limitación entre el TND y la normalidad [2]. Esta última circunstancia está condicionada por la presencia de los síntomas que caracterizan los diferentes TND en la población 'normal'; son la gravedad de estos síntomas, la aparición en edades inapropiadas, la persistencia en el tiempo o la pobre compensación de éstos los que van a vincularse a la discapacidad adaptativa del paciente y a su tipificación como trastorno.

En las últimas clasificaciones internacionales, estos trastornos habían quedado englobados dentro del apartado 'trastornos del comportamiento y de las emociones de comienzo habitual en la infancia y adolescencia' y 'trastornos del desarrollo psicológico' [3] o como 'trastornos de inicio en la infancia, la niñez o la adolescencia' [4]. El término 'trastornos del neurodesarrollo' aparece explícitamente en la última edición del Manual diagnóstico y estadístico de los trastornos mentales (DSM-5), publicada el 18 de mayo de 2013 [5]; se describe un grupo de condiciones (Tabla I) con inicio en el periodo de desarrollo, que se manifiestan de forma temprana - generalmente antes de la entrada en la escuela- y se caracterizan por déficits del desarrollo que producen alteraciones en el adecuado funcionamiento personal, social, académico u ocupacional.

\section{Etiología. Genética}

La totalidad de los TND pueden tener un origen ambiental, genético o, probablemente, multifactorial. En el primer apartado, podemos observar por ejemplo que numerosos TND pueden vincularse a la exposición al alcohol durante la gestación [6]. Sin embargo, tanto la estructura como el funcionamiento cerebral, fuera de la influencia de los factores ambientales, están codificados en la arquitectura genética del individuo. Aberraciones genéticas (mu-
Sección de Neurología Infantil; Hospital Universitario Quirón Madrid (A. Fernández-Jaén, D. Martín Fernández-Mayoralas, A.L. FernándezPerrone). Departamento de Genómica y Medicina; NIMGenetics (J.C. Cigudosa, J. Suela). Departamento de Citogenética Molecular; Centro Nacional de Investigaciones Oncológicas (J.C. Cigudosa). Centro de Salud Doctor Cirajas; Atención Primaria (B. Calleja-Pérez). Departamento de Psicología Biológica y de la Salud; Facultad de Psicología; Universidad Autónoma de Madrid (S. LópezMartín). Madrid, España.

Correspondencia: Dr. Alberto Fernandez Jaén. Sección de Neurología Infantil. Servicio de Neurología. Hospital Universitario Quirón Madrid. Diego de Velázquez, 1. E-28024 Pozuelo de Alarcón (Madrid).

E-mail:

aferjaen@telefonica.net

Declaración de intereses: Los autores manifiestan la inexistencia de conflictos de interés en relación con este artículo.

Aceptado tras revisión externa: 10.01.14.

Cómo citar este artículo: Fernández-Jaén A, Cigudosa JC, Martín Fernández-Mayoralas $D$, Suela J, Fernández-Perrone AL, Calleja-Pérez B, et al. Genética aplicada a la práctica clínica en trastornos del neurodesarrollo. Rev Neurol 2014; 58 (Supl 1): S65-70.

(C) 2014 Revista de Neurología 
Tabla I. Trastornos del neurodesarrollo según el DSM-5 [5].

Discapacidad intelectual

Trastornos de la comunicación

Trastornos del espectro autista

Trastorno por déficit de atención/hiperactividad

Trastorno específico del aprendizaje

Trastornos motores

Otros trastornos del neurodesarrollo

Tabla II. Utilidad del diagnóstico genético en los trastornos del neurodesarrollo.

Tipificación etiológica

Evitación de pruebas médicas innecesarias

Descarte de hipótesis alternativas

Consejo genético familiar

Anticipación pronóstica

Estudio o prevención de afectación multisistémica

Anticipación terapéutica: avances

taciones, deleciones o duplicaciones) que afecten genes necesarios para un correcto funcionamiento del sistema nervioso central pueden manifestarse a través de diferentes TND (formas 'sindrómicas'); en otras ocasiones, la suma de diferentes factores ambientales y la combinación de variantes genéticas normales en la población pueden predisponer al individuo a padecer estos trastornos (formas idiopáticas', probablemente poligénicas) [7]. La elevada frecuencia de manifestaciones sistémicas, rasgos dismórficos -a veces tenues- y fenotipos clínicopronósticos característicos en las formas 'sindrómicas' obliga al especialista al estudio biomédico en los TND, al menos en aquellas formas graves [8].

Las evidencias científicas sobre la contribución genética en la etiopatogenia de los TND es incuestionable y no pretendemos abordarla [9-12]. La heredabilidad de los TND es elevada; se han registrado índices del 0,77-0,88 en entidades tan diferentes como los trastornos del espectro autista (TEA) [13], la dislexia [14] o el trastorno por déficit de atención/ hiperactividad (TDAH) [15]. Los avances en la genética no sólo apoyan el carácter dimensional y categórico de un trastorno [7], sino también el carácter polisintomático de una misma anomalía genética. Así, podemos registrar que diferentes variantes $o$ alteraciones genéticas pueden predisponer a distintos TND [16]; en estudios poblacionales se ha podido demostrar que diferentes regiones o loci genéticos estaban relacionados con una mayor predisposición a TEA, dislexia o TDAH. A través del análisis de la presencia de variaciones en el número de copias (CNV) se han podido identificar variaciones raras o de novo en autismo, esquizofrenia y TDAH; muchas de estas CNV, bien sean deleciones o duplicaciones, afectan a genes implicados en el neurodesarrollo y se vinculan indistintamente a los diagnósticos señalados o a la discapacidad intelectual [17]. En la categorización diagnóstica a través del estudio de gemelos, se ha podido igualmente demostrar una elevada correlación genética entre diferentes TND [18]. Así, una misma CNV patológica puede detectarse en un paciente con TEA y en uno de los padres o hermanos con un perfil clínico diferente (epilepsia, TDAH, discapacidad intelectual, TDAH o esquizofrenia); esta circunstancia o la gravedad del propio trastorno podría estar parcialmente justificada por factores epigenéticos o los modelos de doble impacto [19].

A pesar del conocimiento cada vez más amplio de los factores genéticos en el desarrollo de los TND, la protocolización de los estudios genéticos en el abordaje de los TND queda relegada generalmente a los TEA y la discapacidad intelectual [20-22]. En estos casos, con la entrada de nuevas técnicas, se tipifica su origen genético hasta en el 30\% de ellos [23]. Con independencia de la utilidad de los estudios genéticos en el consejo familiar, la monitorización de manifestaciones sistémicas -muchas de ellas graves- propias de numerosos trastornos monogénicos justifican ampliamente su indicación [22] (Tabla II). Paradójicamente, incluso en los trastornos señalados, numerosos protocolos relegan la conveniencia de los estudios genéticos atendiendo a la historia del paciente, la presentación clínica, la presencia de retraso mental o la historia familiar $[8,24]$. Sin embargo, el hallazgo de aberraciones cromosómicas de novo y, por tanto, la ausencia de antecedentes familiares positivos, el descubrimiento de anomalías genéticas en niños sin retraso mental, la ausencia de rasgos dismórficos y la presencia de una historia médica anodina son frecuentes en estas genopatías.

En el mismo sentido, numerosos trastornos monogénicos o síndromes genéticos pueden manifes- 
tarse por otros TND (trastornos del lenguaje, TDAH, trastornos del desarrollo de la coordinación...) $[25,26]$. La mayor parte de estudios genéticos relacionados con los TND se realizan sobre la discapacidad intelectual, los TEA y el TDAH [27]; los estudios genéticos sobre otros TND son escasos y la protocolización de algoritmos genético-diagnósticos sobre estos TND sin retraso mental o el TDAH es prácticamente inexistente en la literatura médica.

\section{Trastornos del espectro autista. Discapacidad intelectual}

El análisis genético es una práctica habitual en la discapacidad intelectual o el TEA de origen desconocido, pero no siempre protocolizado. Las guías publicadas en las últimas tres décadas han estado marcadas por el empleo de cariotipos de alta resolución, estudios de deleciones subteloméricas o el cribado de trastornos de gen único (X frágil, síndrome de Rett...). Sin embargo, la aparición de técnicas de mayor resolución como los microarrays cromosómicos (CMA) se ha postulado recientemente como la técnica genética de primera línea para estos trastornos [28,29]. Los CMA incluyen todo tipo de análisis del número de copias genómicas basadas en array: la hibridación comparativa genómica (array-CGH) o el array por polimorfismos de nucleótido único (SNP). Los CMA aportan una rentabilidad del 15-30\% en el diagnóstico etiológico de la discapacidad intelectual o los TEA. En los TEA, la rentabilidad diagnóstica es superior en los TEA 'complejos', es decir, cuando presentan otros hallazgos como microcefalia, epilepsia, anomalías congénitas o rasgos dismórficos [29]. En contrapartida, los CMA no detectan reordenamientos equilibrados o mosaicismos de bajo nivel, si bien estas anomalías justifican menos del 1\% de los TND [28].

Este rédito baja considerablemente con el empleo de otras técnicas como el cariotipo convencional, que no supera el 3\%. Indudablemente, el cariotipo o los estudios específicos de trastornos monogénicos deben prevalecer ante la sospecha de síndromes tipificables. El cariotipo debe plantearse ante la sospecha de un síndrome de Down o un perfil clínico compatible con monosomías o trisomías completas, especialmente si se sospechan aneuploidías sexuales (síndrome de Turner, XYY, XXY...); debe descartarse el síndrome $X$ frágil en todo varón con discapacidad intelectual o TEA, o al menos en aquellos con fenotipo característico, historia de TND ligados al X, o antecedentes familiares de insuficiencia ovárica prematura, ataxia o temblores
Figura. Algoritmo de evaluación genética en los trastornos del espectro autista, aplicable a la discapacidad intelectual $[22,29]$.

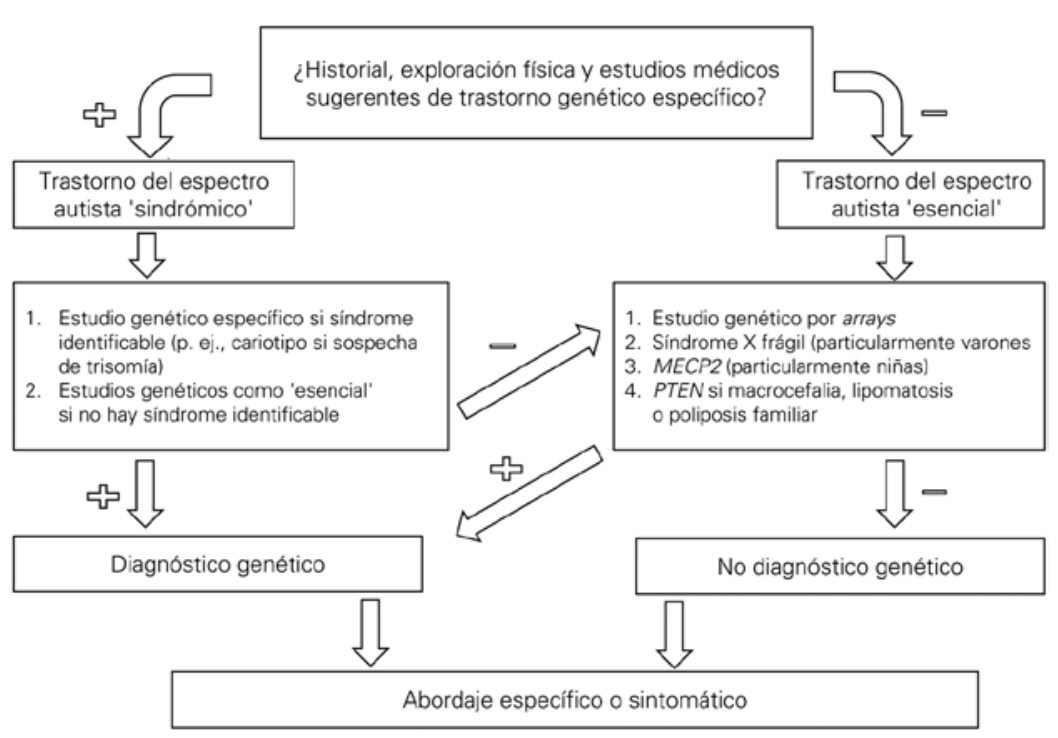

Tabla III. Propuesta de evaluación genética o derivación al genetista en el trastorno por déficit de atención/hiperactividad (a valorar en otros trastornos del neurodesarrollo) [44].

Presencia de rasgos de atipicidad en la clínica (estereotipias, graves problemas en la pragmática del lenguaje...) Asociación con graves problemas de coordinación motora o del lenguaje

Asociación con trastorno de la comunicación social

Cociente intelectual $<70$

Antecedente personal de malformaciones sistémicas (cardíacas, endocrinológicas, óseas...)

Historia familiar (primer o segundo grado) de trastornos del espectro autista, retraso mental, trastornos graves del lenguaje, epilepsia o esquizofrenia

Presencia de rasgos dismórficos

Presencia de macrocefalia o microcefalia ( $\pm 2,5$ desviaciones estándares)

Presencia de talla muy alta o muy baja ( $₫ 2,5$ desviaciones estándares)

$[29,30]$. Del mismo modo, deben contemplarse los estudios mutacionales de MECP2 y PTEN; el primero, al menos en niñas (no exclusivo) con discapacidad intelectual o TEA y microcefalia, y el segundo, especialmente ante la presencia de macrocefalia (> 2,5 desviaciones estándares) [29] o ante- 
cedentes de poliposis intestinal, lipomatosis o trastornos tiroideos [31]. La figura recoge someramente un algoritmo de evaluación diagnóstica y genética aplicable a los TEA o a la discapacidad intelectual.

Debe igualmente señalarse la reciente aparición de las técnicas de secuenciación masiva de nueva generación (entre ellas, la secuenciación exómica), que probablemente podrán resolver o aclarar el origen genético en casos donde los estudios previamente señalados han arrojado resultados normales [32]. Aunque se muestra como una técnica enormemente prometedora, con ratios diagnósticos superiores a los datos señalados, tampoco está exenta de desventajas: falsos positivos, identificación de nuevas variantes difíciles de interpretar, ausencia de protocolos...

\section{Trastorno por déficit de atención/hiperactividad. Otros}

En el conocido carácter poligénico de otros TND se ha justificado la pobre rentabilidad de los estudios genéticos en estos casos. Evaluando con detalle el TDAH, al haber sido en las últimas dos décadas el trastorno más analizado desde la genética [27], podemos observar que la mayor parte de estudios referencian patrones de heredabilidad [33] o variantes genéticas que si bien están presentes en la población general, se muestran con mayor frecuencia en pacientes con TDAH $[11,33]$. La arquitectura genética del TDAH es compleja. La mayor parte de los genes estudiados o analizados codifican transportadores dopaminérgicos, receptores catecolaminérgicos, enzimas metabolizadores de catecolaminas, factores o receptores neurotróficos o proteínas vinculadas a la plasticidad y regulación sináptica. Los polimorfismos de un solo nucleótido referidos en la bibliografía tienen una magnitud de efecto débil y explican una pequeña porción del efecto genético. El análisis futuro de estas variantes, su interacción y su relación con variables sintomáticas, cognitivas o anatómicas, pueden explicar quizá la heterogeneidad de este trastorno y ayudar a establecer estrategias de seguimiento y tratamiento más individualizadas [11].

Sin embargo, el desarrollo y empleo de los CMA ha demostrado en el TDAH, la presencia de microdeleciones o microduplicaciones que afectan a genes previamente involucrados en otros TND (TEA o discapacidad intelectual), epilepsia o esquizofrenia [17]. La frecuencia de estas CNV es más elevada en los pacientes con TDAH que en la población general, y marcadamente más frecuentes ante la aso- ciación un cociente intelectual menor de 70 [34]. Así mismo, el TDAH y otros TND pueden estar presentes en numerosos trastornos monogénicos o síndromes tipificables [26, 35-43] sin la presencia de retraso mental asociado. A la vista de estos datos más actuales, y la relevancia clínica (multisistémica) y pronóstica de estos hallazgos, parece razonable recomendar evaluación genética o derivación al departamento de genética, particularmente en ciertas circunstancias (Tabla III) [44].

\section{Conclusiones}

Los análisis genéticos y el consejo adecuado en los TND van a permitir un mayor y mejor conocimiento de estos problemas. Con independencia de su valor en la tipificación diagnóstica, permitirán un asesoramiento individualizado en el patrón de recurrencia y la posible anticipación y abordaje de enfermedades multisistémicas, algunas de ellas de particular gravedad, especialmente en las formas 'sindrómicas'.

La formación de los especialistas que atienden estos problemas es absolutamente necesaria en este sentido. El diagnóstico etiológico y genético, además de poder ofrecer explicaciones al problema del paciente y evitar teorías de pobre sustento científico, pueden aportar una información vital para el paciente y su familia.

En este sentido, nos enfrentamos con marcada perplejidad al entendimiento de la etiopatogenia de otros TND, entendidos socialmente como 'menos graves' (TDAH, trastornos de la comunicación...). La presencia de ciertos polimorfismos genéticos en estos trastornos podrá aportar una información interesante, quizá en un futuro próximo. A la vista de los últimos hallazgos encontrados con técnicas genéticas de mayor resolución en pacientes con estos trastornos, se insinúa relevante el abordaje genético, particularmente en las formas 'sindrómicas' de estos TND 'menos graves'.

Bibliografía

1. Goldstein S, Reynolds CR. Handbook of neurodevelopmental and genetic disorders in children. 2 ed. New York: Guilford Press; 2011.

2. Artigas-Pallarés J, Narbona J. Trastornos del neurodesarrollo. Barcelona: Viguera; 2011.

3. Organización Panamericana de la Salud. Clasificación estadística internacional de enfermedades y problemas relacionados con la salud: CIE-10. 10 ed. Washington DC: Organización Panamericana de la Salud; 2003.

4. American Psychiatric Association. Diagnostic criteria from DSM-IV-TR. Washington DC: APA; 2000.

5. American Psychiatric Association. Diagnostic and statistical 
manual of mental disorders, fifth edition: DSM-5. Washington DC: APA; 2013.

6. Martín Fernández-Mayoralas D, Fernández-Jaén A. Fetopatía alcohólica: puesta al día. Rev Neurol 2011; 52 (Supl 1): S53-7.

7. Artigas-Pallarés J, Guitart M, Gabau-Vila E. Bases genéticas de los trastornos del neurodesarrollo. Rev Neurol 2013; 56 (Supl 1): S23-34.

8. Díez-Cuervo A, Muñoz-Yunta JA, Fuentes-Biggi J, Canal-Bedia R, Idiazábal-Aletxa MA, Ferrari-Arroyo MJ, et al. Guía de buena práctica para el diagnóstico de los trastornos del espectro autista. Rev Neurol 2005; 41: 299-310.

9. Betancur C. Etiological heterogeneity in autism spectrum disorders: more than 100 genetic and genomic disorders and still counting. Brain Res 2011; 1380: 42-77.

10. Neale BM, Medland SE, Ripke S, Asherson P, Franke B, Lesch KP, et al. Meta-analysis of genome-wide association studies of attention-deficit/hyperactivity disorder. J Am Acad Child Adolesc Psychiatry 2010; 49: 884-97.

11. Fernández-Jaén A, Martín Fernández-Mayoralas D, CallejaPérez B, Muñoz-Jareño N, López-Arribas S. Endofenotipos genómicos del trastorno por déficit de atención/hiperactividad. Rev Neurol 2012; 54 (Supl 1): S81-7.

12. Ropers HH. Genetics of early onset cognitive impairment Annu Rev Genomics Hum Genet 2010; 11: 161-87.

13. Hallmayer J, Cleveland S, Torres A, Phillips J, Cohen B, Torigoe T, et al. Genetic heritability and shared environmental factors among twin pairs with autism. Arch Gen Psychiatry 2011; 68: 1095-102.

14. Astrom RL, Wadsworth SJ, DeFries JC. Etiology of the stability of reading difficulties: the longitudinal twin study of reading disabilities. Twin Res Hum Genet 2007; 10: 434-9.

15. Larsson H, Chang Z, D'Onofrio BM, Lichtenstein P. The heritability of clinically diagnosed attention deficit hyperactivity disorder across the lifespan. Psychol Med 2013; Oct 10. [Epub ahead of print].

16. Fernández-Jaén A, Martín Fernández-Mayoralas D, Fernández-Perrone AL, Calleja-Pérez B, Muñoz-Jareño N, López-Arribas S. Autismo y trastorno por déficit de atención/ hiperactividad: intervención farmacológica. Rev Neurol 2013; 57 (Supl 1): S205-10.

17. Elia J, Gai X, Xie HM, Perin JC, Geiger E, Glessner JT, et al. Rare structural variants found in attention-deficit hyperactivity disorder are preferentially associated with neurodevelopmental genes. Mol Psychiatry 2010; 15: 637-46.

18. Lichtenstein P, Carlstrom E, Rastam M, Gillberg C, Anckarsater H. The genetics of autism spectrum disorders and related neuropsychiatric disorders in childhood. Am J Psychiatry 2010; 167: 1357-63.

19. Maynard TM, Sikich L, Lieberman JA, LaMantia AS. Neural development, cell-cell signaling, and the 'two-hit' hypothesis of schizophrenia. Schizophr Bull 2001; 27: 457-76.

20. Díaz-Anzaldua A, Díaz-Martínez A. Contribución genética, ambiental y epigenética en la susceptibilidad a los trastornos del espectro autista. Rev Neurol 2013; 57: 556-68.

21. Flore LA, Milunsky JM. Updates in the genetic evaluation of the child with global developmental delay or intellectual disability. Semin Pediatr Neurol 2012; 19: 173-80.

22. Carter MT, Scherer SW. Autism spectrum disorder in the genetics clinic: a review. Clin Genet 2013; 83: 399-407.

23. Willemsen M, Kleefstra T. Making headway with genetic diagnostics of intellectual disabilities. Clin Genet 2013; Jul 29. [Epub ahead of print].

24. Isaksen J, Bryn V, Diseth TH, Heiberg A, Schjolberg S, Skjeldal $\mathrm{OH}$. Children with autism spectrum disorders -the importance of medical investigations. Eur J Paediatr Neurol 2013; 17: 68-76.

25. Jones KL, Smith DW. Patrones reconocibles de malformaciones humanas. 6 ed. Madrid: Elsevier; 2007.
26. O'Brien G. Behavioural phenotypes in clinical practice. London: Mac Keith; 2002.

27. Bishop DV. Which neurodevelopmental disorders get researched and why? PLoS One 2010; 11: e15112.

28. Miller DT, Adam MP, Aradhya S, Biesecker LG, Brothman AR, Carter NP, et al. Consensus statement: chromosomal microarray is a first-tier clinical diagnostic test for individuals with developmental disabilities or congenital anomalies. Am J Hum Genet 2010; 86: 749-64.

29. Schaefer GB, Mendelsohn NJ; Professional Practice and Guidelines Committee. Clinical genetics evaluation in identifying the etiology of autism spectrum disorders: 2013 guideline revisions. Genet Med 2013; 15: 399-407.

30. Lubs HA, Stevenson RE, Schwartz CE. Fragile X and X-linked intellectual disability: four decades of discovery. Am J Hum Genet 2012; 90: 579-90.

31. Lachlan KL, Lucassen AM, Bunyan D, Temple IK. Cowden syndrome and Bannayan Riley Ruvalcaba syndrome represent one condition with variable expression and age-related penetrance: results of a clinical study of PTEN mutation carriers. J Med Genet 2007; 44: 579-85.

32. Ostrer $\mathrm{H}$. Changing the game with whole exome sequencing. Clin Genet 2011; 80: 101-3.

33. Faraone SV, Perlis RH, Doyle AE, Smoller JW, Goralnick JJ, Holmgren MA, et al. Molecular genetics of attention-deficit/ hyperactivity disorder. Biol Psychiatry 2005; 57: 1313-23.

34. Williams NM, Zaharieva I, Martin A, Langley K, Mantripragada K, Fossdal R, et al. Rare chromosomal deletions and duplications in attention-deficit hyperactivity disorder: a genome-wide analysis. Lancet 2010; 376: 1401-8.

35. Fernández-Jaén A, Martín Fernández-Mayoralas D, MuñozJareño N, Izquierdo L, Calleja-Pérez B. Síndrome de BannayanRiley-Ruvalcaba: a propósito de un caso. Acta Pediatr Esp 2007; 65: 519-23.

36. Martín Fernández-Mayoralas D, Fernández-Jaén A, MuñozJareño N, Melón M, Pleguezuelos M, Calleja-Pérez B. Aspectos clínicos de tres pacientes con el síndrome 48,XXYY. Acta Pediatr Esp 2010; 68: 197-201.

37. Prontera P, Bernardini L, Stangoni G, Capalbo A, Rogaia D, Romani R, et al. Deletion 2p15-16.1 syndrome: case report and review. Am J Med Genet A 2011; 155A: 2473-8.

38. Portnoi MF, Lebas F, Gruchy N, Ardalan A, Biran-Mucignat V, Malan V, et al. 22q11.2 duplication syndrome: two new familial cases with some overlapping features with DiGeorge/ velocardiofacial syndromes. Am J Med Genet A 2005; 137 : 47-51.

39. De Boer L, Roder I, Wit JM. Psychosocial, cognitive, and motor functioning in patients with suspected Sotos syndrome: a comparison between patients with and without NSD1 gene alterations. Dev Med Child Neurol 2006; 48: 582-8.

40. Tang SX, Yi JJ, Calkins ME, Whinna DA, Kohler CG, Souders MC, et al. Psychiatric disorders in 22q11.2 deletion syndrome are prevalent but undertreated. Psychol Med 2013; Sep 9. [Epub ahead of print].

41. Nahata L, Rosoklija I, Yu RN, Cohen LE. Klinefelter syndrome: are we missing opportunities for early detection? Clin Pediatr (Phila) 2013; 52: 936-41.

42. Deshpande PS, Coffey DB. Fragile X syndrome and attentiondeficit/hyperactivity disorder symptoms. J Child Adolesc Psychopharmacol 2011; 21: 639-42.

43. Kraan CM, Hocking DR, Georgiou-Karistianis N, Metcalfe SA, Archibald AD, Fielding J, et al. Impaired response inhibition is associated with self-reported symptoms of depression, anxiety, and ADHD in female FMR1 premutation carriers. Am J Med Genet B Neuropsychiatr Genet 2014; 165: 41-51.

44. Fernández-Jaén A. TDAH y sindromología. In Soutullo C, ed. TDAH IV. Barcelona: Instituto de Psicofarmacología del Niño y Adolescente; 2013. p. 113-26. 


\section{Genetics applied to clinical practice in neurodevelopmental disorders}

Summary. The medical literature contains a wide body of evidence supporting genetic involvement in neurodevelopmental disorders. Advances made in genetics and technology have increased the diagnostic cost-effectiveness of current studies from $3-5 \%$ to $30-40 \%$ in patients with intellectual disability or autism spectrum disorders. In this regard, chromosomal microarray studies display greater diagnostic power than conventional techniques (karyotype, subtelomeric analyses, etc.). The latest protocols in the biomedical field of the genetic study of these disorders cite chromosomal microarrays as the first-line analysis, while also recommending other specific studies depending on the patient's clinical features (fragile $\mathrm{X}$ syndrome, PTEN mutation, etc.). In the evaluation of other neurodevelopmental disorders (attention deficit hyperactivity disorder, learning disorders, etc.), the number of genetic tests carried out is limited and conditioned by the clinical characteristics or the patient's familial or personal history. Even in these situations, there are no genetic referral or evaluation protocols.

Key words. Arrays. Attention deficit hyperactivity disorder. Autism. Genetics. Intellectual disability. Neurodevelopmental disorders. 